\title{
BMJ Open Beyond forest plots: clinical gestalt and its influence on COPD telemonitoring studies and outcomes review
}

\author{
Sheree M Smith (D) , ${ }^{1,2}$ Anne E Holland, ${ }^{2,3,4}$ Christine F McDonald ${ }^{2,5}$
}

To cite: Smith SM, Holland AE, McDonald CF. Beyond forest plots: clinical gestalt and its influence on COPD telemonitoring studies and outcomes review. BMJ Open 2019;9:e030779. doi:10.1136/ bmjopen-2019-030779

- Prepublication history and additional material for this paper are available online. To view these files, please visit the journal online (http://dx.doi. org/10.1136/bmjopen-2019030779).

Received 01 April 2019

Revised 20 November 2019

Accepted 20 November 2019

Check for updates

(c) Author(s) (or their employer(s)) 2019. Re-use permitted under CC BY-NC. No commercial re-use. See rights and permissions. Published by BMJ.

${ }^{1}$ School of Nursing and Midwifery, Western Sydney University, Penrith South, New South Wales, Australia ${ }^{2}$ Institute for Breathing and Sleep, Austin Health, Melbourne Victoria, Australia

${ }^{3}$ Department of Allergy, Immunology and Respiratory Medicine, Monash University, Melbourne, Victoria, Australia ${ }^{4}$ Department of Physiotherapy, Alfred Health, Melbourne,

Victoria, Australia

${ }^{5}$ Department of Respiratory and Sleep Medicine, Austin Hospital, Heidelberg, Victoria, Australia

Correspondence to Professor Sheree M Smith; Sheree.Smith@westernsydney. edu.au

\section{ABSTRACT}

Background Chronic obstructive pulmonary disease (COPD) is a progressive chronic condition. Improvements in therapies have resulted in better patient outcomes. The use of technology such as telemonitoring as an additional intervention is aimed at enhancing care and reducing unnecessary acute hospital service use. The influence of verbal communication between health staff and patients to inform decision making regarding use of acute hospital services within telemonitoring studies has not been assessed.

Method A systematic overview of published systematic reviews of COPD and telemonitoring was conducted using an a priori protocol to ascertain the impact of verbal communication in telemonitoring studies on health service outcomes such as emergency department attendances, hospitalisation and hospital length of stay. The search of the following electronic databases: Cochrane Library, Medline, Pubmed, CINAHL, Embase, TROVE, Australian Digital Thesis and Proquest International Dissertations and Theses was conducted in 2017 and updated in September 2019.

Results Six systematic reviews were identified. All reviews involved home monitoring of COPD symptoms and biometric data. Included reviews reported 5-28 studies with sample sizes ranging from 310 to 2891 participants. Many studies reported in the systematic reviews were excluded as they were telephone support, cost effectiveness studies, and/or did not report the outcomes of interest for this overview. Irrespective of group assignment, verbal communication with the health or research team did not alter the emergency attendance or hospitalisation outcome. The length of stay was longer for those who were assigned home telemonitoring in the majority of studies.

Conclusion This overview of telemonitoring for COPD had small sample sizes and a wide variety of included studies. Communication was not consistent in all included studies. Understanding the context of communication with study participants and the decision-making process for referring patients to various health services needs to be reported in future studies of telemonitoring and COPD.

\section{INTRODUCTION}

Chronic obstructive pulmonary disease (COPD) is a progressive condition associated with symptoms of dyspnoea, cough and fatigue as well as recurrent 'flare ups'

\section{Strengths and limitations of this study}

Telemonitoring may be useful for chronic obstructive pulmonary disease patients who do not have easy access to acute clinical care.

- Identification of a lack of detail associated with the communication between the participant and the research team raises the issue of study quality and interpretation of health services outcomes.

- The systematic reviews utilised in this overview reported studies with small numbers of participants.

- Interpretation of an overview is challenging however the lack of data reporting communication with the research or clinical team is concerning.

or exacerbations, which may necessitate hospitalisation. ${ }^{1}$ Hospitalisations are associated with a more rapid loss of lung function and increased mortality as well as impacting adversely on the quality of life of people with COPD. ${ }^{1-3}$ Improvements in care of patients with COPD have been attributed to both pharmacological and non-pharmacological interventions. ${ }^{4}$ Of the latter, the use of health technology in the form of telemonitoring has been explored, with the aims of detecting and intervening early in exacerbations and, ideally, reducing hospitalisations and healthcare costs. $^{5}$

Telemonitoring is defined as 'the use of telecommunication technologies by patients for the timely transmission of data such as spirometric measures, vital signs and symptoms from home to a health care service centre' (Jaana et al, p313). ${ }^{6}$ One focus of COPD telemonitoring studies has been to serve as a prompt for health professionals to start a dialogue with the patient regarding their clinical status and thereby to direct care. ${ }^{6}$ The directing care aspect of technology has raised issues of reliance on the health professional to review the data in a timely manner and to respond with, at the very least, a clinical review of the patient. ${ }^{7}$ The incorporation of a face-to-face response to telecommuted 
patient data has been proven to be effective in nonCOPD-specific settings where patient access to a clinician is increased for those living in isolated environments such as rural and remote areas. ${ }^{89}$ For the majority of COPD patients who live within metropolitan and urban areas where clinical services are available and COPD care is consistent with best practice, reviews suggest the benefit of telemonitoring is limited. ${ }^{10} 11$

In systematic reviews ${ }^{12-14}$ of telemonitoring, heterogeneity of technological applications has been found and these reviews have also identified methodological concerns with some studies. The systematic review process uses a structured procedure for evaluating the inherent quality of a study through risk of bias assessment including subject selection, allocation concealment, outcome blinding and attrition assessment. ${ }^{15} 16$ When evidence of efficacy is inconsistent in different systematic reviews, a systematic review of systematic reviews or 'systematic overview of reviews' may be helpful. ${ }^{17}{ }^{18}$ In a systematic review of telemonitoring in COPD by Bolton $e t a l^{12}$ it was suggested that the benefit of telemonitoring per se in the absence of other care packages, was not proven. However, the influence of human communication reported within studies may not have been assessed. ${ }^{11}{ }^{19}$ Studies of online or telephone-based triage systems for out of hours health advice have found patients are often sent to the emergency department as a risk averse strategy ${ }^{20}$ as it is the safest decision in the absence of more robust clinical data. Whether the addition of telemonitoring enhances communication and improves decision-making is unclear. Communication between the patient and clinician may contribute to clinician decision making in response to patient reports of changing symptoms and the need for varying clinical care. This approach to clinical reasoning is incorporated in the descriptor 'clinical gestalt'. 2223

To better understand the effect of COPD telemonitoring we sought to investigate the potential influence and impact of verbal communication between clinical and/ or research staff and patients on health service outcomes in COPD telemonitoring studies. We hypothesised that verbal communication between health professionals and patients in telemonitoring studies can influence outcomes.

\section{METHODS}

We performed a systematic overview ${ }^{24}$ of COPD telemonitoring reviews published within the last 7 years, incorporating two central objectives. First, we sought to identify from published systematic reviews, reported communication within telemonitoring studies that resulted in any form of clinical medical review of patients. Second, we assessed the impact of communication and the efficacy of the COPD telemonitoring in terms of health utilisation outcomes of studies reported within systematic reviews.

Systematic reviews with and without meta-analysis of trials of telemonitoring in COPD were identified through electronic database searches. The data of studies reported within these selected reviews were probed regarding episodes of verbal communication between clinical and/ or research staff and COPD patients, irrespective of group assignment, to evaluate the potential effect patient-staff communication may have had on study outcomes.

\section{Patient and public involvement}

All data for this review were from published studies. Patients and the members of the public who may have been involved in the original research were not part of this overview of systematic reviews.

\section{Search strategy}

An a priori protocol was developed and implemented, reflecting current best practice guidance ${ }^{25}$ for undertaking systematic reviews. A systematic search of peerreviewed literature using electronic databases was performed in accordance with Preferred Reporting Items for Systematic Reviews and Meta-Analyses ${ }^{26}$ and systematic review of systematic reviews guidance. ${ }^{17}$ From May to August 2017, we searched the following electronic databases: Cochrane Library, Medline, Pubmed, Cumulative Index to Nursing and Allied Health Literature, Embase, TROVE, Australian Digital Thesis and Proquest International Dissertations and Theses. References of retrieved publications were scanned for any additional citations such as government sponsored health technology reviews. "Telemonitoring" OR Tele* AND "review" AND "COPD" search terms were combined (see online supplementary table 1: search strategy example). Search limits included systematic reviews published in the English language and publication date within 7 years to ensure the most up-todate study results were being reviewed. An updated search was subsequently completed in September 2019.

\section{Eligibility criteria}

Publications were eligible for inclusion if they were systematic reviews with and without meta-analyses reporting on the efficacy of monitoring people with COPD through the use of telemonitoring. Systematic reviews were considered if they were consistent with Cochrane systematic review methodology and reported an assessment of bias. ${ }^{15}$ The participants of interest were adults (18 years and over) who had medically diagnosed COPD and were electronically monitored at home. Once reviews were identified, we subsequently examined each study reported within the selected reviews to ascertain the technology used and the outcome measurements recorded. We utilised Jaana $e t a l$ 's definition of telemonitoring as 'the use of telecommunication technologies by patients for the timely transmission of data (eg, spirometric measures, vital signs and symptoms) from home to a health care service centre' (Jaana et al, p313) ${ }^{6}$ for selection of both systematic reviews and published studies within included reviews for predetermined analysis. Studies that related to only telephone support were excluded.

Telehealth and telemedicine studies and reviews may have broader definitions in that the technology's wide 

healthcare consultations to professional education. These studies and reviews were excluded from this overview. ${ }^{27}$ Case studies, commentary papers, narrative reviews and case report series were also excluded. Of interest was evidence of clinical review. For this systematic overview, a clinical review was defined as any conversation between a patient and the clinical or research staff that involved any aspect of clinical care or treatment. The primary outcome was acute care hospital health service utilisation comprising hospital admission, emergency department attendances and length of hospital stay. Studies included in the analyses were stratified by reported clinical communication in control and intervention groups.

For this systematic overview, a predetermined two-step procedure for identification and selection of systematic reviews and subsequent selection and extraction of data from individual studies reported within selected reviews was determined prior to commencement. After database searches were conducted, duplicate records were removed, inclusion criteria were applied and the eligibility assessment of the retrieved systematic reviews application ranges from direct clinical care such as remote

\section{Systematic review selection and individual studies data extraction}

was completed. Inclusion criteria comprised COPD telemonitoring systematic reviews related specifically to home-based electronic monitoring where COPD patients entered their biometric data and the information was telecommuted to a clinical service. All reviews that pertained only to specific telehealth interventions such as video conferencing, electronic self-management education and disease management, and telephone support were excluded.

Two independent reviewers (SMS, AEH) scanned titles and reviewed abstracts for relevance. Full-text systematic reviews were obtained for further assessment and application of the inclusion criteria. Any disagreements over inclusion of systematic reviews were resolved through discussion and consensus was reached. An independent arbitrator (CFM) was available should consensus not be reached (figure 1).

In the second step of this overview, all studies that were reportedly utilised in the meta-analyses in the included reviews were identified and full text articles were obtained as not all studies in the systematic reviews met our definition of telemonitoring. ${ }^{6}$ The unit of analysis was the individual study rather than the systematic review. Studies were included in the meta-analysis only if we were able

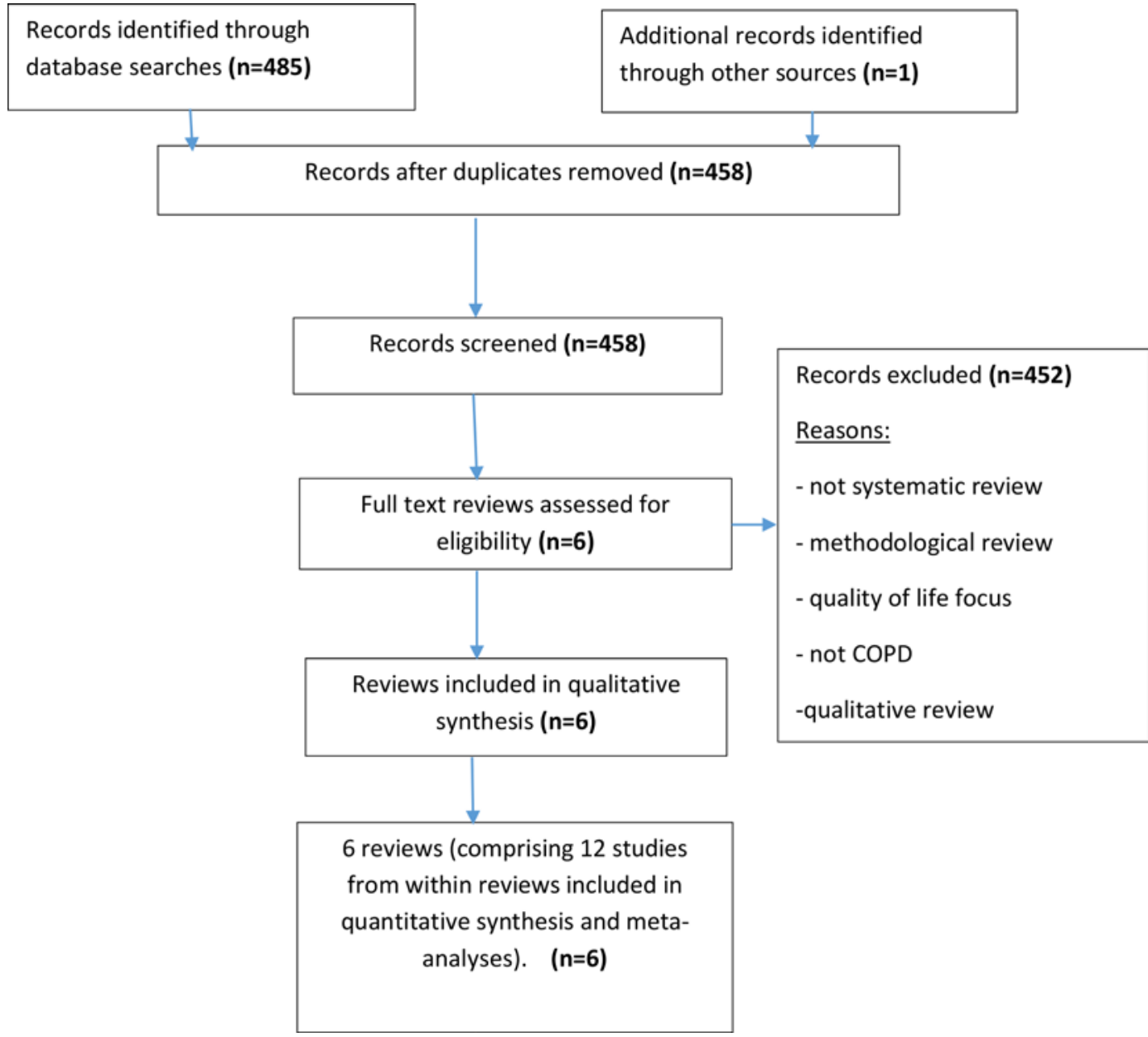

Figure 1 Flow diagram for selection of COPD telemonitoring reviews. Source: Moher et al. ${ }^{26}$ COPD, chronic obstructive pulmonary disease. 


\begin{tabular}{cl} 
Table 1 & AMSTAR rating scales questions for assessing methodological quality of systematic reviews * \\
\hline No. & Question and response scale yes; no; can't answer; not applicable \\
\hline 1 & Was an 'a priori' design provided? \\
3 & Was there duplicate study selection and data extraction? \\
4 & Was a comprehensive literature search performed? \\
5 & Was the status of publication (ie, grey literature) used as an inclusion criterion? \\
6 & Was a list of studies (included and excluded) provided? \\
7 & Were the characteristics of the included studies provided? \\
8 & Was the scientific quality of the included studies assessed and documented? \\
9 & Was the scientific quality of the included studies used appropriately in formulating conclusions? \\
10 & Were the methods used to combine the findings of studies appropriate? \\
11 & Was the likelihood of publication bias assessed?
\end{tabular}

*From: Shea et al. BMC Medical Research Methodology 2007;7:10. doi:10.1186/1471-2288-7-10.

to identify communication with the research or clinical team, in order to enable answering our a priori question. Each full text article was scanned for described episodes of verbal communication between study participants and clinical or research team members. Data extraction was performed using a predetermined report form. Health utilisation outcome data and identification of communication in either control or intervention group for each study were recorded.

\section{Data availability statement}

All data relevant to the study are included in the article or uploaded as supplementary information and all data were from published studies reported in published systematic reviews.

\section{Systematic review quality assessment}

An assessment of the methodological quality of the selected reviews was undertaken to ensure only highquality data were available to be analysed. The Assessing Methodological Quality of Systematic Reviews (AMSTAR) guidance $^{28}$ was utilised for the methodological quality assessment (table 1).

Two reviewers assessed the quality of eligible systematic reviews utilising AMSTAR checklist (SMS, AEH) and assigned a rating to each selected systematic review. ${ }^{28}$ Assessment of individual study methodological quality within the selected systematic reviews was not undertaken as this assessment had been performed as part of the original systematic review process and was reported within each included review.

\section{Data synthesis and analysis}

Data from included studies from within included systematic reviews were pooled and a priori data synthesis and analyses of healthcare utilisation data were undertaken. Two comparisons were examined:

1. Studies to evaluate the combined impact of telemonitoring and clinical communication where the telemonitoring intervention group had clinical communication and control group had no reported contact with the clinical or research team apart from standard outpatient appointments.

2. Studies to evaluate the impact of telemonitoring alone, over and above the effects of communication that reported participants communicating with either study staff or clinicians in both control and intervention groups.

Analyses were limited to health services utilisation outcomes of interest: hospitalisation, emergency department attendance and length of hospital stay. Data were combined using RevMan V.5.3 software. We used fixed effect ORs for variables such as counts of emergency department attendance and hospital admission. For length of stay data (continuous variable) a fixed effect standard mean difference was utilised. In these metaanalyses, heterogeneity was considered and random effect models utilised when heterogeneity was considered to be substantial. Heterogeneity was measured by the percentage of variation across studies and reported as the $\mathrm{I}^{2}$ statistic; if heterogeneity was greater than $50 \%$ this reflected substantial heterogeneity (Higgins and Green, p278). ${ }^{15}$

\section{RESULTS}

Four hundred and eighty-three (483) records were retrieved with additional records being identified through hand searching. The total number of records retrieved was 458 after duplicate records were removed. Titles and abstracts were obtained and eligibility criteria applied resulting in 452 publications being considered ineligible. Six systematic reviews were suitable for inclusion in this systematic overview. Reasons for exclusion comprised methodological reviews which concentrated on the telemonitoring methods used, participants not having COPD, only quality of life outcomes, not meeting 
Table 2 Overview of included systematic reviews

\begin{tabular}{|c|c|c|c|c|}
\hline $\begin{array}{l}\text { Review } \\
\text { year }\end{array}$ & Aim (participants) & Search strategy & $\begin{array}{l}\text { Studies } \\
\text { included (N) }\end{array}$ & $\begin{array}{l}\text { Total no. of } \\
\text { participants }\end{array}$ \\
\hline $\begin{array}{l}\text { Cruz } \\
2014\end{array}$ & $\begin{array}{l}\text { To assess the effectiveness of } \\
\text { home telemonitoring to reduces } \\
\text { healthcare utilisation and } \\
\text { improve health related outcomes } \\
\text { of patients with COPD. }\end{array}$ & $\begin{array}{l}\text { Medline, Embase, B-online knowledge Library } \\
\text { and Web of Science databases (June-August } \\
\text { 2012) } \\
\text { Search terms provided. } \\
\text { English, Portuguese and Spanish publications. }\end{array}$ & 9 & 587 \\
\hline $\begin{array}{l}\text { Polisena } \\
2010\end{array}$ & $\begin{array}{l}\text { To examine a meta-analysis } \\
\text { of clinical outcomes, patient's } \\
\text { quality of life (QoL) and the use } \\
\text { of healthcare services for home } \\
\text { teleheath compared with those } \\
\text { of usual care (UC) for patients } \\
\text { with COPD. }\end{array}$ & $\begin{array}{l}\text { Ovid interface, PubMed, Cochrane library } \\
\text { and the Centre of reviews and dissemination } \\
\text { databases (1998 onwards) } \\
\text { Search terms provided. } \\
\text { No language restrictions. }\end{array}$ & 9 & 914 \\
\hline $\begin{array}{l}\text { Franek } \\
2012\end{array}$ & $\begin{array}{l}\text { To conduct an evidence-based } \\
\text { assessment of home telehealth } \\
\text { technologies for patients with } \\
\text { COPD. }\end{array}$ & $\begin{array}{l}\text { Ovid Medline, Medline in-process and } \\
\text { other non-indexed citations, EMBASE, the } \\
\text { Cumulative index to Nursing and Allied Health } \\
\text { Literature, the Cochrane Library, International } \\
\text { Agency for Health and Technology Assessment } \\
\text { (1 January 2000-3 November 2010) } \\
\text { No search terms provided. } \\
\text { English publications. }\end{array}$ & 5 & 310 \\
\hline $\begin{array}{l}\text { McLean } \\
2011\end{array}$ & $\begin{array}{l}\text { To review the effectiveness } \\
\text { of telehealth care for COPD } \\
\text { compared with face-to-face } \\
\text { usual care in improving quality } \\
\text { of life and reducing accident and } \\
\text { emergency department visits and } \\
\text { hospitalisations. }\end{array}$ & $\begin{array}{l}\text { Cochrane Airways Group Specialised Register } \\
\text { of trial. } \\
\text { Search (up to January 2010). Search terms } \\
\text { provided. } \\
\text { No language restrictions. }\end{array}$ & 10 & 1004 \\
\hline
\end{tabular}

COPD, chronic obstructive pulmonary disease.

the overview's definition of telemonitoring, qualitative reviews and editorial commentaries on published systematic reviews and the use of telemonitoring technology. Figure 1 illustrates the process of selection of systematic reviews and the subsequent identification of studies.

\section{Overview of included systematic reviews}

A summary of included systematic reviews is provided in table 2. Four of the six systematic reviews reported an absence of language restrictions as part of their search strategy. All reviews involved home monitoring of COPD symptoms and biometric data such as vital signs and spirometric readings. Study participants had previously been diagnosed with COPD and the majority of study participants were 60 years or over with moderate to severe disease. ${ }^{29}$ Included reviews reported between 5 and 28 studies and had sample sizes ranging from 310 to 2891 participants, resulting in a total overview population of
5768 study participants. Many of the systematic reviews included studies of telephone support, ${ }^{30-33}$ cost effectiveness studies that did not report outcome data such as length of stay, hospitalisation or emergency department attendance ${ }^{34}$ and single group ${ }^{35} 36$ studies and these were excluded from our review. In terms of the telemonitoring intervention, any studies reported in the included systematic reviews that did not meet our predetermined definition of telemonitoring such as web-based applications for self-management were also excluded. ${ }^{37}$

\section{Quality of evidence assessment for included reviews}

The assessment of the quality of COPD telemonitoring systematic reviews included in this overview has been tabulated in table 3 . In five systematic reviews a priori protocols were not reported. ${ }^{12-142738}$ Grey literature formed part of the search strategy in three reviews ${ }^{12} 2739$ and reference lists were utilised. Exclusion information was available for 
Table 3 AMSTAR quality of evidence rating for assessment methodological quality of systematic reviews*

\begin{tabular}{ll}
\hline Quality of evidence rating & AMSTAR score \\
\hline Low & $0-4$ \\
Medium & $5-8$ \\
High & $9-11$ \\
\hline
\end{tabular}

*From: Seo H-J et al. ${ }^{40}$

five reviews ${ }^{12-143839}$ with the sixth review reporting exclusion data based solely on study design. One review ${ }^{39}$ had an extensive framework developed specifically for the undertaking of the systematic review that incorporated agreement of definitions associated with the review, an initial development phase using concepts maps and the use of innovative strategies for searching databases and other publication portals. Differences in the quality of reviews are reflected in the AMSTAR scores (range 6-11), indicating various elements not being reported in all reviews, resulting in moderate and high-quality evidence. ${ }^{40}$

\section{Characteristics of included studies}

Table 4 summarises the characteristics of included studies from the systematic reviews that reported communication with patients ${ }^{41-45}$ as well as studies ${ }^{11}$ 46-51 that indicated limited (eg, beginning and end of study) contact with study participants and reported health services outcomes. The included studies $(n=12)$ in this overview had relatively small sample sizes ranging from 40 to 344 participants. Studies were conducted over differing periods of time ranging from 1 to 12 months. Four studies followed participants for 12 months, ${ }^{11} 41434451$ one study for 9 months, ${ }^{47}$ four for 6 months, ${ }^{42454649}$ two for 3 months ${ }^{4850}$ and one study followed up participants at 2 months. ${ }^{19}$ The age of the COPD patient population was similar across all studies with mean age of 69 years for both the home telemonitoring and control groups. The telemonitoring interventions differed across studies and support for participants in control groups also differed between studies (table 4 ).

\section{Effect of telemonitoring interventions on emergency attendance}

Of the included studies in this systematic overview, eight studies $^{1941-4345484951}$ reported emergency attendance as a study outcome. Some studies reported this outcome as median (IQR), ${ }^{45}$ mean and $\mathrm{SD}^{42}{ }^{41}$ without reporting the actual count of events or number of patients attending the emergency department. These studies that did not report event counts or patient numbers were not included in the quantitative synthesis of emergency department attendance data and are reported individually.

Comparison 1: there was no difference in emergency attendances between telemonitoring and usual care groups in three studies ${ }^{19} 4849$ when only telemonitoring group participants were able to communicate with the clinical or research staff $(p=0.39)$ as part of the study protocol (see online supplementary figure 1 ). The two studies ${ }^{42} 51$ which only reported emergency attendance as means (SD) demonstrated no difference between groups. Comparison 2: in studies ${ }^{41}$ where group assignment did not limit participants communicating with the clinical or research team, there were a greater number of emergency department attendances in the usual care group ( $p=0.03$ ) (see online supplementary figure 2 ). In one study ${ }^{45}$ that reported only medians (IQR) for emergency department attendances, the telemonitoring group $0(0,0.08)$ and control group, $0(0.10)$ demonstrated no difference between groups for emergency attendances $(\mathrm{p}=0.24)$ and therefore no conclusions can be drawn in relation to the impact of verbal communication on emergency attendances.

\section{Hospitalisation and telemonitoring}

Hospitalisation was an outcome of studies in nine telemonitoring studies. $111941-4345484951$ Hospitalisation data from two studies ${ }^{1151}$ were excluded from the synthesis as they provided only means and SD and were not sufficient to include in the meta-analysis. In these two studies there was no difference between groups for hospitalisation. The number of hospitalisations reported as events in the remaining seven studies were included in the analysis.

Comparison 1: in the three studies ${ }^{1948} 49$ where communication was limited to the telemonitoring group, usual care participants had more hospital admissions although this result was not statistically significant $(p=0.12)$ (see online supplementary figure 3 ).

Comparison 2: in four studies ${ }^{41-43} 45$ which reported that both usual care and telemonitoring participants were able to communicate with their clinical and/or research teams, more hospital admissions were reported in the usual care group $(p=0.02)$ (see online supplementary figure 4).

\section{Length of stay and telemonitoring}

Length of stay in telemonitoring studies was primarily reported as means and SD to reflect a continuous variable. Length of stay was measured in nine of the included studies. ${ }^{11} 19424345-4749-51$ Two of these studies ${ }^{4647}$ reported the average hospital length of stay and one study ${ }^{45}$ provided medians and IQR for both telemonitoring and control groups. These studies were excluded from analysis due to insufficient data.

Comparison 1: when communication was limited to participant assignment to the telemonitoring intervention, ${ }^{11} 19$ 49-51 there was no difference between groups $(\mathrm{p}=0.76)$ (see online supplementary figure 5$)$. The telemonitoring participants were hospitalised for a longer period of time in three studies. ${ }^{11} 1950$ This finding was consistent with the two studies ${ }^{46} 47$ in which average length of stay was reported with one study ${ }^{47}$ reporting the telemonitoring group's average length of stay as 9.7 days compared with the usual care group having 6.9 days in hospital. In the second study, ${ }^{46}$ the telemonitoring group 


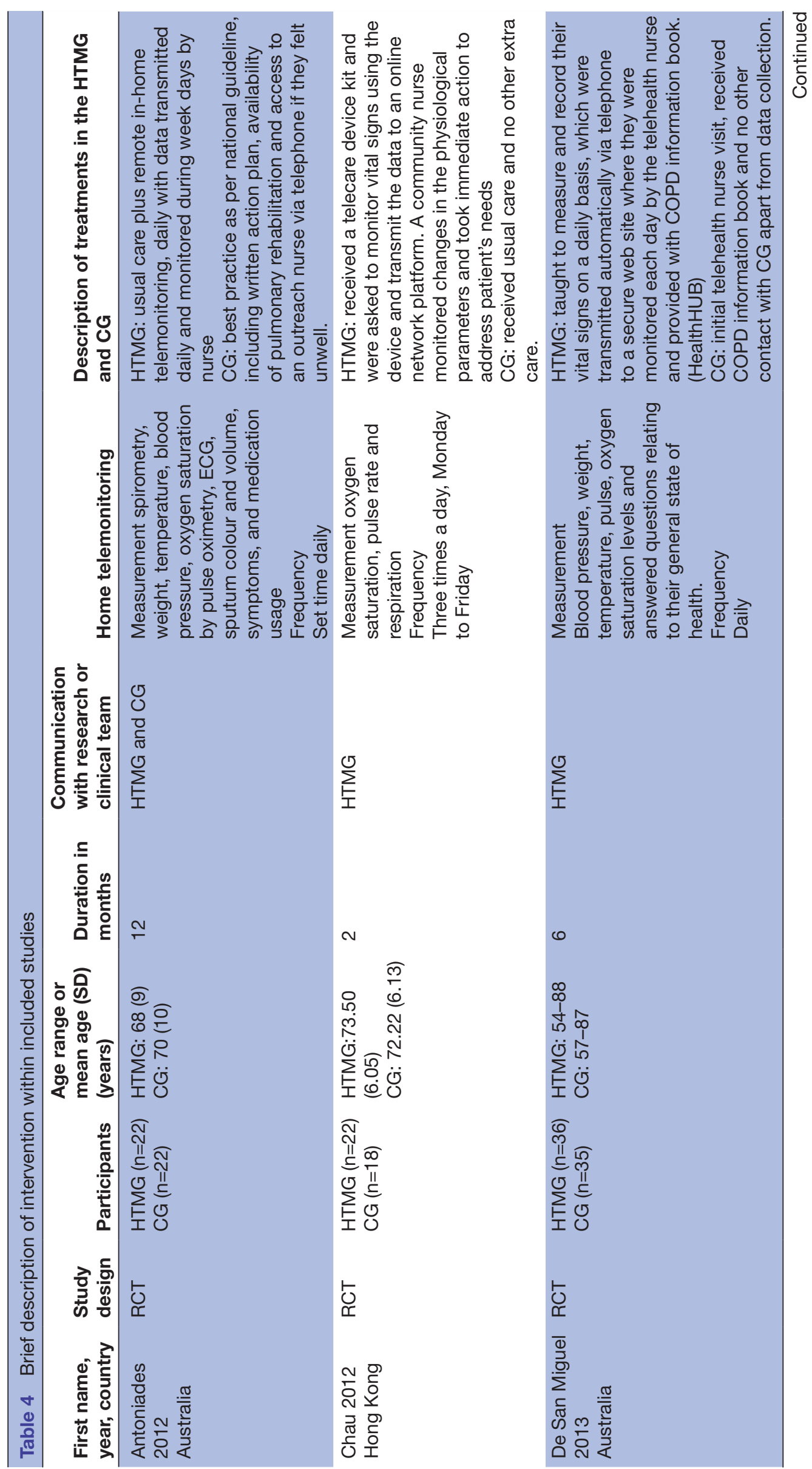




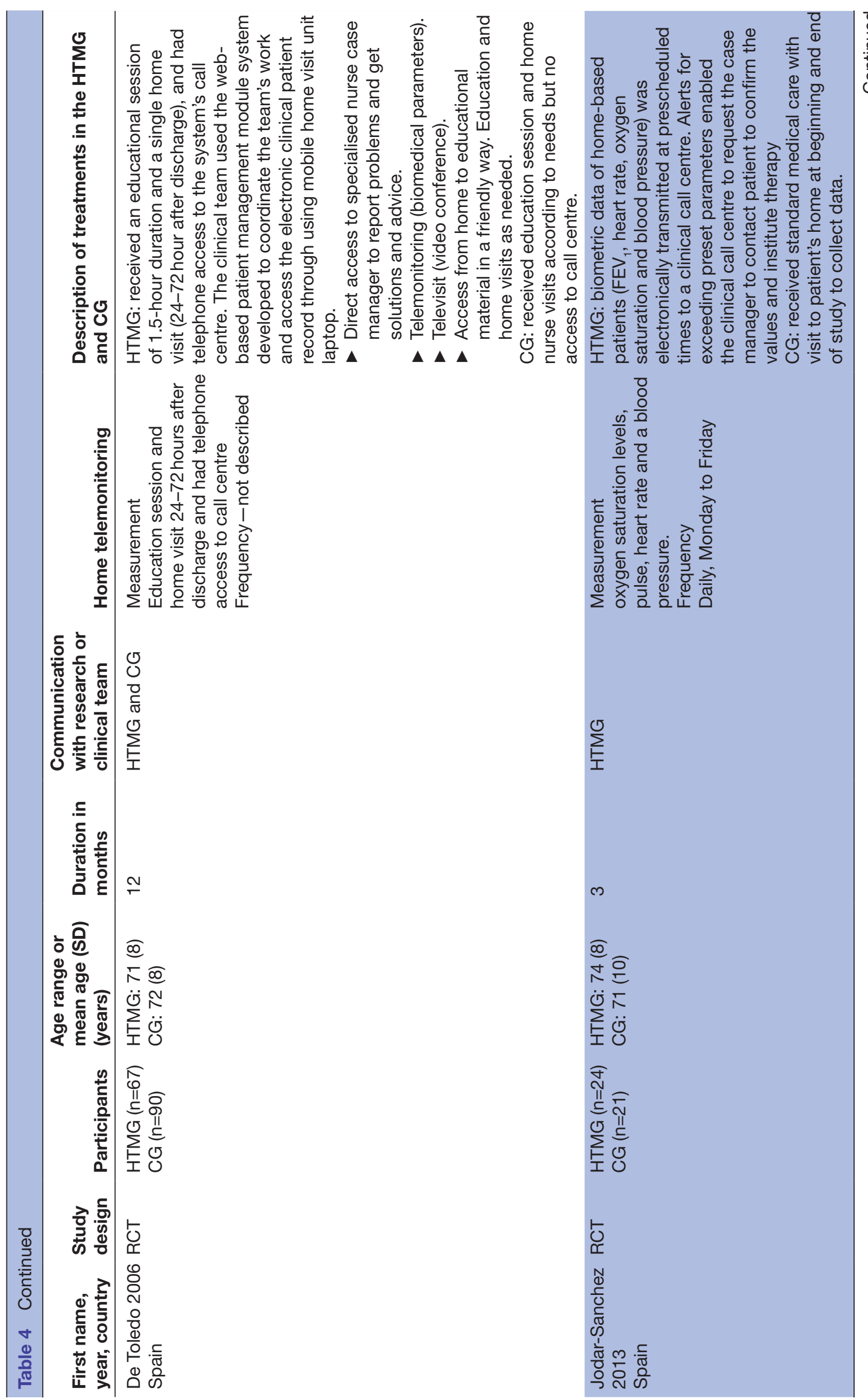




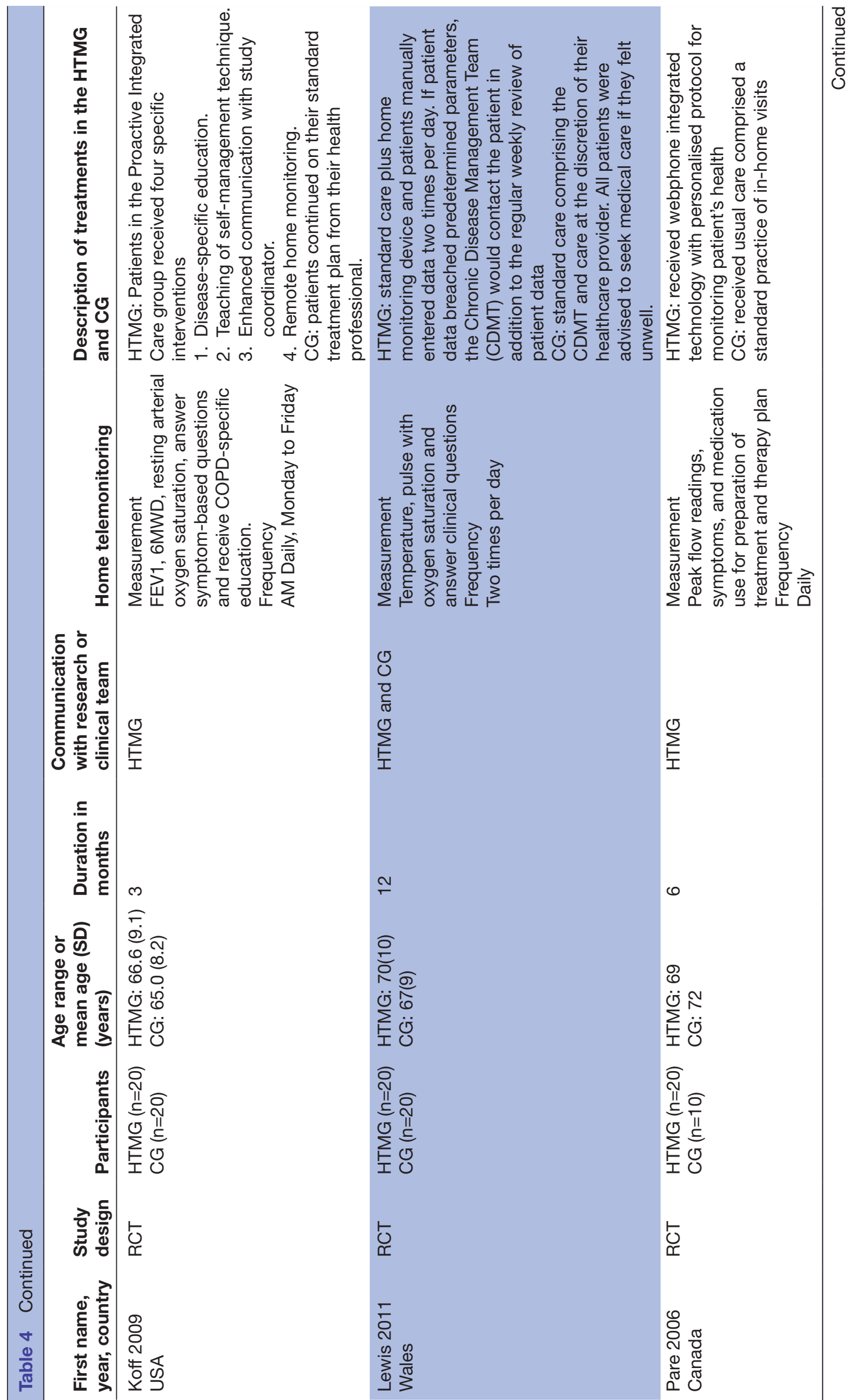




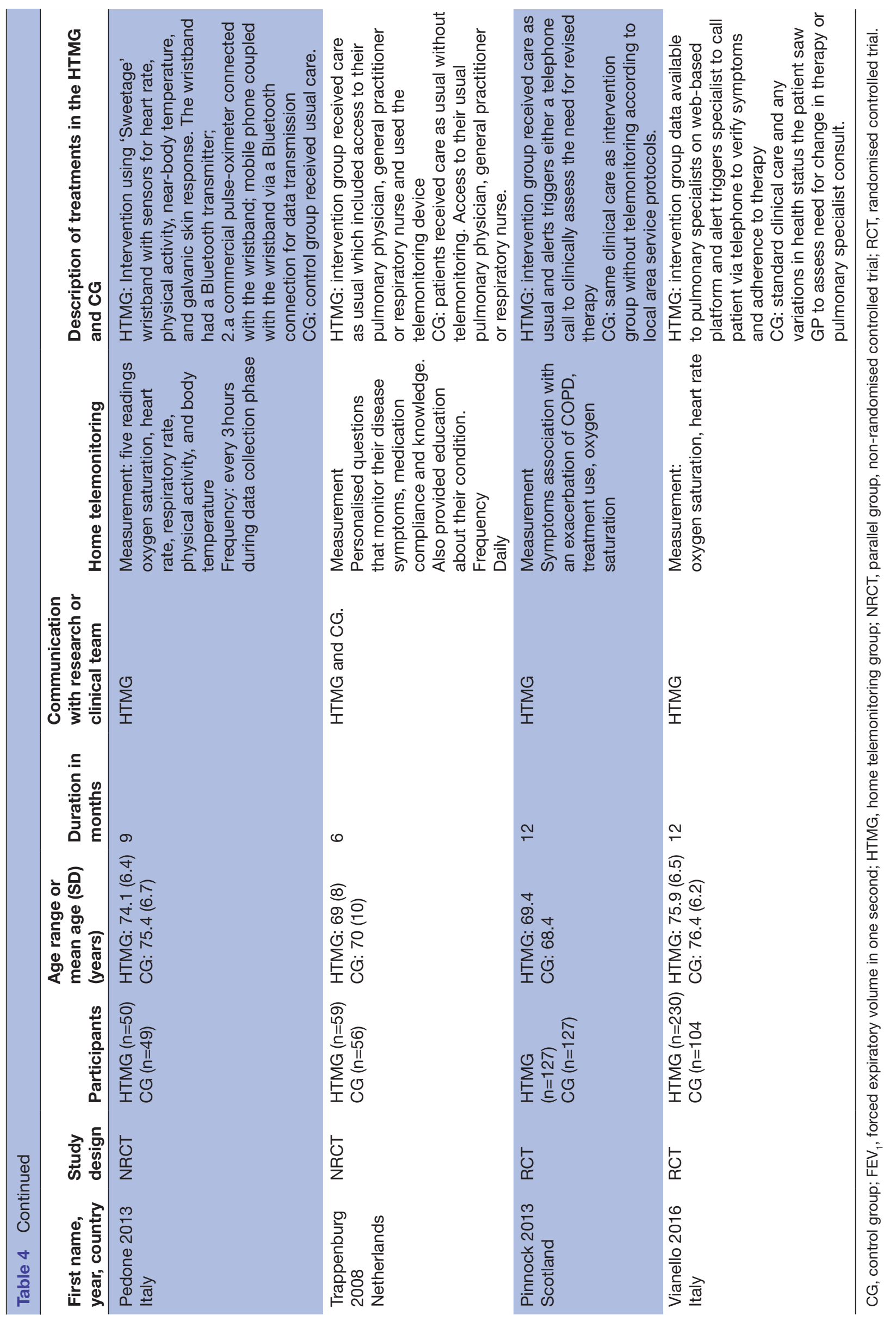


had an average of 13.5 days in hospital while the usual care group had 7.3 days in hospital.

Comparison 2: when study participants were able to communicate with the clinical and/or research team irrespective of group assignment in two studies, ${ }^{42}{ }^{43}$ there was no statistical difference in length of stay between groups $(\mathrm{p}=0.56)$ (see online supplementary figure 6 ). One study ${ }^{45}$ that reported medians and IQR found no difference between groups. This suggests no impact of telemonitoring over and above communication with the health team in relation to length of stay.

\section{DISCUSSION}

Studies of adults communicating symptoms to health professionals in the absence of biometric data (such as through out of hours' care help lines) have shown that such programmes provide risk averse advice resulting in more hospital and emergency admissions. ${ }^{20}{ }^{21}$ This overview of telemonitoring systematic reviews identified differences in health services outcomes for participants who had ongoing access to communication with the clinical or research teams involved in the included clinical trials, compared with those who did not. The nature and context associated with these differences is difficult to ascertain and is beyond the scope of this overview.

When communication was available to study participants regardless of group assignment, there were statistical differences in emergency department attendance with fewer telemonitoring participants seeking emergency care. The context and understanding of the behaviour of seeking or not seeking care from the emergency department remains unclear. It is also unclear if the health services seeking behaviour was directed by the telemonitoring research/clinical team and how this decision was made for study participants. Similarly, it is unclear if the usual care group participants' description of their symptoms when liaising with clinical or research staff, in the absence of biometric data, increased the likelihood of being advised to seek medical review including emergency department attendance. When communication was limited to only the telemonitoring group, it is unknown if the usual care participants sought advice from their general practitioner and were directed to report or selfreported to the emergency department ${ }^{52}$; understanding this behaviour warrants further investigation and detailed reporting.

The pooled hospital admission data confirmed the telemonitoring group had fewer hospital admissions irrespective of the usual care group's ability to communicate with the clinical or research team. The need for hospital care indicates a progression of disease, exacerbation and/or failure of therapy. As the telemonitoring intervention provided biometric data, this clinical surveillance may have assisted institution of earlier outpatient therapy (eg, by study clinician recommendation of action plan implementation or GP or respiratory nursing outreach visit), thus reducing the need for hospitalisation. However, reporting of the data that led to the decision regarding hospitalisation was unclear in all included studies.

A notable finding of this overview was the impact of communication on length of stay in hospital. The usual care group had a shorter hospital stay as compared with the telemonitoring group when communication with the research and or clinical team did not form part of the study protocol. In one study, ${ }^{51}$ patients were assigned 2:1 to the telemonitoring intervention with the pulmonologists caring for telemonitoring group patients having direct access to their telemonitoring data. Pulmonologists could respond to any patient alerts enabling immediate specialist review. This length of stay data synthesis finding presents a challenge to its interpretation, as there are several hypotheses that could account for this finding. Details related to the patient's medical condition were not provided and it may be that the telemonitoring group were more unwell on admission and required a longer length of stay. Furthermore, the relationship between a longer length of stay and disease progression was not provided in the published data. It is unclear if the participants in usual care group adopted an approach to hospitalisation that may have been successful for them in the past. ${ }^{52}$ MacKichan et a $\tilde{l}^{2}$ report patients' experiences of primary care and/or out-of-hours services that led patients to seek medical treatment from the emergency department. Specifically, long wait times for routine appointments, previous experience of out-of-hours care and the belief the EDs could offer specialist level care that was not routinely available in primary care were reported by patients. There is a dearth of information regarding the impact of patient telemonitoring on primary care practitioners' likelihood of referral of patients for ED assessment or hospitalisation, but one could hypothesise that practitioners may refer more readily for ED assessment and/or hospitalisation if their patient is part of a control group for a telemonitoring study. We have limited knowledge about how well-matched the intervention and usual care groups in studies in this review were in respect to disease severity, comorbidities and other potentially important phenotypic differences, which may have impacted their length of hospital stay.

This overview of systematic reviews highlights the limitation of using blunt health services outcome measures when there is a lack of clarity and information pertaining to usual care. ${ }^{11}$ Particularly, the question remains whether usual care was consistent with evidenced based guideline-driven care prior to the commencement of the studies. ${ }^{11}$ Moreover, information associated with the context around the hospital admission such as severity, comorbidity and biometric admission data and clinical decision making parameters may provide a better insight into the utility of interventions such as telemonitoring in the management of COPD care. Clarity around the degree of communication available to all groups that informs decision making and level of access to clinical support will be important for future studies of telemedicine in COPD. 


\section{Limitations}

There are several limitations associated with this overview of systematic reviews. ${ }^{24}$ First, it was inherently difficult to pool data when various studies reported findings using different statistical methods, resulting in inconsistent reporting of outcomes and missing data. Second, standardised reporting of results within interventional studies would be useful for the conduct of meta-analyses within systematic reviews. Finally, in overviews of systematic reviews there is a potential for 'overlap', meaning that the same studies in different reviews could potentially be counted twice. ${ }^{24}$ In this overview, we only pooled data once from individual studies reported in systematic reviews.

\section{CONCLUSION}

This overview of COPD telemonitoring systematic reviews found communication with the clinical and/or research team was not consistent in all studies. The access to support through the ability to communicate with the clinical team may have impacted on health service outcomes. Further research is required to distill the extent of the impact on outcome measures particularly when participants assigned to usual care have limited access to support.

\section{Twitter Sheree M Smith @shereesmi}

Contributors SMS conceived the study, all authors contributed to defining and refining the review question and scope of the review. SMS, CMD and AEH evaluated published systematic reviews for methodological quality and within each review, study data of episodes of verbal communication with patients as a form of clinical review. All authors reviewed the findings and contributed to the content, writing, drafting and revision of the manuscript, and agreed to the final version.

Funding The authors have not declared a specific grant for this research from any funding agency in the public, commercial or not-for-profit sectors.

Competing interests None declared.

Patient consent for publication Not required.

Provenance and peer review Not commissioned; externally peer reviewed.

Data availability statement Data are available in a public, open access repository. There are no data in this work. Data are available upon reasonable request. Data may be obtained from a third party and are not publicly available. All data relevant to the study are included in the article or uploaded as supplementary information.

Open access This is an open access article distributed in accordance with the Creative Commons Attribution Non Commercial (CC BY-NC 4.0) license, which permits others to distribute, remix, adapt, build upon this work non-commercially, and license their derivative works on different terms, provided the original work is properly cited, appropriate credit is given, any changes made indicated, and the use is non-commercial. See: http://creativecommons.org/licenses/by-nc/4.0/.

ORCID iD

Sheree M Smith http://orcid.org/0000-0002-7469-1022

\section{REFERENCES}

1 Donaldson GCet al. Relationship between exacerbation frequency and lung function decline in chronic obstructive pulmonary disease. Thorax 2002;57:847-52.

2 Carrasco Garrido P, de Miguel Díez J, Rejas Gutiérrez J, et al. Negative impact of chronic obstructive pulmonary disease on the health-related quality of life of patients. Results of the EPIDEPOC study. Health Qual Life Outcomes 2006;4:31.
3 Seemungal TA, Donaldson GC, Paul EA, et al. Effect of exacerbation on quality of life in patients with chronic obstructive pulmonary disease. Am J Respir Crit Care Med 1998;157:1418-22.

4 Yang I, Dabscheck E, George J, et al. The COPD-X plan: Australian and New Zealand guidelines for the management of chronic obstructive pulmonary disease, version 2.44, 2015. Available: http:// copdx.org.au/copd-x-plan/ [cited 3 Mar 2016].

5 Henderson C, Knapp M, Fernández J-L, et al. Cost effectiveness of telehealth for patients with long term conditions (whole systems Demonstrator telehealth questionnaire study): nested economic evaluation in a pragmatic, cluster randomised controlled trial. BMJ 2013;346:f1035.

6 Jaana M, Paré G, Sicotte C. Home telemonitoring for respiratory conditions: a systematic review. Am J Manag Care 2009;15:313.

7 Smith SM, Recruitment ESL. Challenges and disparities associated with Telemonitoring as part of COPD supported discharge study: patients and carers and clinical staff perspectives. Am J Respir Crit Care Med 2010;181:A5975.

8 Heaney D, Caldow J, McClusky C, et al. The introduction of a new consulting technology into the National health service (NHS) for Scotland. Telemed J E Health 2009;15:546-51.

9 Yellowlees PM, Odor A, Parish MB, et al. A feasibility study of the use of asynchronous telepsychiatry for psychiatric consultations. Psychiatric Services 2010;61:838-40.

10 Fodgren G, Rachas A, Farmer AJ, et al. Interactive telemedicine: effects on professional practice and health care outcomes. Cochrane Database Syst Rev 2015.

11 Pinnock H, Hanley J, McCloughan L, et al. Effectiveness of telemonitoring integrated into existing clinical services on hospital admission for exacerbation of chronic obstructive pulmonary disease: researcher blind, multicentre, randomised controlled trial. BMJ 2013;347:f6070.

12 Bolton CE, Waters CS, Peirce S, et al. Insufficient evidence of benefit: a systematic review of home telemonitoring for COPD. J Eval Clin Pract 2011;17:1216-22.

13 Polisena J, Tran K, Cimon K, et al. Home telehealth for chronic obstructive pulmonary disease: a systematic review and metaanalysis. J Telemed Telecare 2010;16:120-7.

14 Cruz J, Brooks D, Marques A. Home telemonitoring effectiveness in COPD: a systematic review. Int J Clin Pract 2014;68:369-78.

15 Higgins JPT, Green S. Cochrane Handbook for systematic reviews of interventions. The Cochrane Collaboration 2011;5.1. [updated March 2011].

16 Pandor A, Thokala P, Gomersall T, et al. Home telemonitoring or structured telephone support programmes after recent discharge in patients with heart failure: systematic review and economic evaluation. London: National Insitute of Health Research, 2013.

17 Smith V, Devane D, Begley CM, et al. Methodology in conducting a systematic review of systematic reviews of healthcare interventions. BMC Med Res Methodol 2011;11:1-6.

18 Bobrovitz N, Onakpoya I, Roberts N, et al. Protocol for an overview of systematic reviews of interventions to reduce unscheduled hospital admissions among adults: Table 1. BMJ Open 2015;5:e008269.

19 Chau JP-C, Lee DT-F, Yu DS-F, et al. A feasibility study to investigate the acceptability and potential effectiveness of a telecare service for older people with chronic obstructive pulmonary disease. Int J Med Inform 2012;81:674-82.

20 Huibers L, Smits M, Renaud V, et al. Safety of telephone triage in out-of-hours care: a systematic review. Scand J Prim Health Care 2011;29:198-209.

21 Semigran HL, Linder JA, Gidengil C, et al. Evaluation of symptom checkers for self diagnosis and triage: audit study. BMJ 2015;351.

22 Kabrhel C, Camargo CA, Goldhaber SZ. Clinical gestalt and the diagnosis of pulmonary embolism: does experience matter? Chest 2005;127:1627-30.

23 Cook C. Is clinical gestalt good enough? J Man Manip Ther 2009;17:6-7.

24 McKenzie JE, Brennan SE. Overviews of systematic reviews: great promise, greater challenge. Syst Rev 2017;6:185.

25 Moher D, Shamseer L, Clarke M et al. Preferred reporting items for systematic review and meta-analysis protocols (PRISMA-P) 2015 statement. Syst Rev 2015;4:1-9.

26 Moher D, Liberati A, Tetzlaff J, et al. Preferred reporting items for systematic reviews and meta-analyses: the PRISMA statement. PLoS Med 2009;6:e1000097.

27 Franek J. Home telehealth for patients with chronic obstructive pulmonary disease (COPD): an evidence-based analysis. Ontario Health Technology Assessment Series 2012;12:1-58.

28 Shea BJ, Hamel C, Wells GA, et al. AMSTAR is a reliable and valid measurement tool to assess the methodological quality of systematic reviews. J Clin Epidemiol 2009;62:1013-20. 
29 Vestbo J, Hurd SS, Agustí AG, et al. Global strategy for the diagnosis, management, and prevention of chronic obstructive pulmonary disease. Am J Respir Crit Care Med 2013;187:347-65.

30 Wong KW, Wong FKY, Chan MF. Effects of nurse-initiated telephone follow-up on self-efficacy among patients with chronic obstructive pulmonary disease. J Adv Nurs 2005;49:210-22.

31 Bourbeau J, Julien M, Maltais F, et al. Reduction of hospital utilization in patients with chronic obstructive pulmonary disease: a disease-specific self-management intervention. Arch Intern Med 2003;163:585-91.

32 Chandler MH, Clifton GD, Louis BA, et al. Home monitoring of theophylline levels: a novel therapeutic approach. Pharmacotherapy 1990;10:294-300.

33 Sorknaes AD, Madsen $\mathrm{H}$, Hallas J, et al. Nurse tele-consultations with discharged COPD patients reduce early readmissions - an interventional study. Clin Respir J 2011;5:26-34.

34 Vitacca M, Bianchi L, Guerra A, et al. Tele-assistance in chronic respiratory failure patients: a randomised clinical trial. European Respiratory Journal 2009;33:411-8.

35 Vontetsianos T, Giovas P, Katsaras T, et al. Telemedicine-assisted home support for patients with advanced chronic obstructive pulmonary disease: preliminary results after nine-month follow-up. $J$ Telemed Telecare 2005;11:86-8.

36 Maiolo C, Mohamed El, Fiorani CM, et al. Home telemonitoring for patients with severe respiratory illness: the Italian experience. $J$ Telemed Telecare 2003;9:67-71.

37 Nguyen HQ, Donesky-Cuenco D, Wolpin S, et al. Randomized controlled trial of an Internet-based versus face-to-face dyspnea self-management program for patients with chronic obstructive pulmonary disease: pilot study. J Med Internet Res 2008;10:e9.

38 Sul A-R, Lyu D-H, Park D-A. Effectiveness of telemonitoring versus usual care for chronic obstructive pulmonary disease: a systematic review and meta-analysis. $J$ Telemed Telecare 2018;11:1357633X1881175.

39 McLean S, Sheikh A, Cresswell K, et al. The impact of Telehealthcare on the quality and safety of care: a systematic overview. PLoS One 2013;8:e71238.

40 Seo H-J, Kim KU. Quality assessment of systematic reviews or metaanalyses of nursing interventions conducted by Korean reviewers. BMC Med Res Methodol 2012;12:129.
41 deToledo P, Jimeanez S, delPozo F, et al. Telemedicine experience for chronic care in COPD. IEEE Trans. Inform. Technol. Biomed. 2006;10:567-73.

42 Trappenburg JCA, Niesink A, de Weert-van Oene GH, et al. Effects of telemonitoring in patients with chronic obstructive pulmonary disease. Telemed J E Health 2008;14:138-46.

43 Antoniades NC, Rochford PD, Pretto JJ, et al. Pilot study of remote Telemonitoring in COPD. Telemedicine and e-Health 2012;18:634-40.

44 Casas Aet al. Integrated care prevents hospitalisations for exacerbations in COPD patients. European Respiratory Journal 2006;28:123-30.

45 Lewis KE, Annandale JA, Warm DL, et al. Does home Telemonitoring after pulmonary rehabilitation reduce healthcare use in optimized COPD?? A pilot randomized trial. COPD 2011;7:44-50.

46 Paré G, Sicotte C, St.-Jules D, et al. Cost-Minimization analysis of a Telehomecare program for patients with chronic obstructive pulmonary disease. Telemedicine and e-Health 2006;12:114-21.

47 Pedone C, Chiurco D, Scarlata S, et al. Efficacy of multiparametric telemonitoring on respiratory outcomes in elderly people with COPD: a randomized controlled trial. BMC Health Serv Res 2013;13.

48 Koff PB, Jones $\mathrm{RH}$, Cashman JM, et al. Proactive integrated care improves quality of life in patients with COPD. European Respiratory Journal 2009;33:1031-8.

49 De San Miguel K, Smith J, Lewin G. Telehealth remote monitoring for community-dwelling older adults with chronic obstructive pulmonary disease. Telemedicine and e-Health 2013;19:652-7.

50 Jódar-Sánchez F, Ortega F, Parra C, et al. Implementation of a telehealth programme for patients with severe chronic obstructive pulmonary disease treated with long-term oxygen therapy. J Telemed Telecare 2013;19:11-17.

51 Vianello A, Fusello M, Gubian L, et al. Home telemonitoring for patients with acute exacerbation of chronic obstructive pulmonary disease: a randomized controlled trial. BMC Pulm Med 2016;16:157

52 MacKichan F, Brangan E, Wye L, et al. Why do patients seek primary medical care in emergency departments? An ethnographic exploration of access to general practice. BMJ Open 2017;7:e013816. 